\title{
Factores de Riesgo para Endometriosis
}

\author{
INSTITUTO DE CIENCIAS DE LA SALUD - CES PROFAMILIA, MEDELLIN
}

Dra. Adriana María Cuartas Calle*

\section{INTRODUCCION}

La endometriosis es una entidad en la cual el epitelio y el estroma característicos del endometrio, proliferan en sitios diferentes al cuerpo del útero, pero usualmente en la pelvis.

En los últimos años se ha visto un aumento en la frecuencia de endometriosis. Algunos autores reconocen que este incremento puede ser debido a mejores métodos diagnósticos y otros lo interpretan como un aumento real. La verdadera prevalencia de endometriosis pélvica no se conoce debido a la ausencia de estudios sobre la población general. Botero y colaboradores, estudiaron los informes laparoscópicos desde 1979 hasta septiembre de 1984 y encontraron endometriosis en el $2.5 \%$ de las lapaparoscopias diagnósticas y en el 1.21 por mil de las tubectomías laparoscópicas en Profamilia de Medellín Colombia (1).

La patogénesis de la endometriosis es desconocida. Aunque se han formulado muchas hipótesis, aún no existe un modelo definido que explique su etiología. Las teorías clásicas implican los siguientes mecanismos: la teoría de Sampson de la menstruación retrógrada y la implantación, en la cual, las células endometriales son regurgitadas a través de las trompas hasta su localización patológica $(2,3)$, la metaplasia o desarrollo del tejido endometrial ectópico que surge in situ a partir de la diferenciación anormal del epitelio germinal $(4,5,6,7,8)$ y alguna combinación de estas teorías $(9,10,11)$.

* Gineco-Obstetra CES., Gineco-Obstetra HGM.
Para explicar el raro hallazgo de lesiones endometriósicas en localizaciones extrabdominales, existe la hipótesis formulada por Halban, de metástasis benignas a través del sistema hematológico y linfático (12).

Actualmente, los investigadores intentan determinar el posible papel etiológico de los factores inmunológico y endocrino. La teoría inmunológica está sustentada por la presencia del factor C3 del complemento en el endometrio uterino de todas las mujeres con endometriosis (10), la demostración de disminución de la respuesta inmunológica mediada por células a los antígenos endometriales autólogos $(9,13,14)$ y más recientemente, Barbieri y colaboradores encontraron aumento del $\mathrm{Ca}-125$ en las mujeres con endometriosis estadios III y IV, lo cual apoya la teoría inmunológica, por ser el Ca-125 un determinante antigénico definido por el anticuerpo monoclonal OC-125 $(15,16)$. Con respecto a desórdenes endocrinos, se ha descrito endometriosis asociada con: Hiperprolactinemia $(15,17,18)$, defectos en la Fase lútea $(15,19)$ y síndrome de Folículo lutenizado no roto $(20,21,22)$. También se ha informado endometriosis asociada a un aumento en la incidencia de aborto espontáneo (23).

Desde el punto de vista epidemiológico, pocas variables se han encontrado asociadas con endometriosis. Se considera edad de riesgo para endometriosis toda la vida reproductiva (25). Para el grupo adolescente se ha descrito aumento del riesgo cuando existe anomalía uterina que obstruye el flujo menstrual $(24,25)$. Un estudio descriptivo realizado en Profamilia de Medellín, encontró mavor ries- 
go para dos grupos de edad: el de 25-29 años en donde se presentaron el $30.3 \%$ y el de $30-34$ años en donde se halló el 28.2\% de los casos (1). Con respecto a la raza, se ha dicho que la endometriosis es más frecuente en las mujeres de raza blanca, pero una revisión publicada recientemente hace notar que el riesgo se incrementa en las mujeres de raza negra si su estado socioeconómico es alto (25). También se menciona que existe mayor riesgo para endometriosis entre las mujeres con nivel socioeconómico alto $(25,26)$ y especialmente en aquellas que postergan la maternidad (25).

Existen publicaciones que mencionan otras características que también se relacionan con el desarrollo de la endometriosis. Una preponderancia familiar entre madres y hermanas de mujeres con endometriosis ha sido demostrada por Simpson, sugiriendo un componente genético para la producción de la enfermedad (27). Existe la evidencia de que el endometrio menstrual es viable y es capaz de crecer en cultivo tisular (28), cuando es transplantado a otras zonas del cuerpo en animales de experimentación $(29,30)$ y cuando es implantado directamente en incisiones quirúrgicas $(31,32,33)$. Esto último, se ha descrito después de lesión vesical (31), posterior a ligadura tubárica $(32,33)$, luego de conización (34), en periné después de episiotomía y en cicatriz de cesárea. Se cree que el implante endometriósico por vía retrógrada, puede favorecerse en las mujeres con obstrucción del tracto genital bajo $(24,35,36)$, en las mujeres con hipermenorrea y polimenorrea en quienes se aumenta el tiempo de exposición pélvica al producto menstrual (37) y en las usuarias de tampón rutinariamente (38). Un trabajo reciente, menciona dos hechos que pueden actuar como protectores o inhibidores de la formación de endometriosis. Ellos son: el ejercicio como disciplina adquirida desde edad temprana y el hábito de fumar cigarrillo como característica adquirida antes de los 17 años y con consumo de más de 20 cigarrillos por día (37). Igualmente, hay sitios en los cuales se ha informado una tendencia a la disminución en la prevalencia de endometriosis, paralelamente al aumento en las ventas de anovulatorios orales (9).

A pesar de la alta frecuencia de esta enfermedad, las características de las mujeres con riesgo para desarrollar endometriosis no han sido examinadas sistemáticamente por métodos epidemiológicos. El estudio de factores de riesgo contribuye a enten- der mejor la etiología de la enfermedad, ayuda a enfocar el diagnóstico y a establecer medidas preventivas.

En este estudio, se pretende investigar si la endometriosis es más frecuente en mujeres con: edad menor de 20 años y edad comprendida entre 30 y 50 años, raza blanca, estado socieconómico alto, profesional, antecedentes familiares de endometriosis, edad temprana de la menarquia, baja paridad, edad tardía del primer embarazo, ciclo menstrual con hipermenorrea y polimenorrea, anomalía uterina que obstruye el flujo menstrual, poste rior a implante quirúrgico y en usuarias de tampón rutinariamente. También se pretende averiguar si la endometriosis es menos frecuente en las mujeres que tienen el hábito de fumar cigarrillo, practican ejercicio rutinariamente y en las usuarias de anticonceptivos orales. Además, se quiere buscar que asociación existe entre endometriosis y el uso de dispositivo intrauterino.

Con el propósito de evaluar cuál es la asociación existente entre los posibles factores de riesgo y la presencia de la enfermedad, se analizará por separado cada variable comparando los casos conlos tres tipos diferentes de controles.

\section{MATERIAL Y METODOS}

Se realizó ùn estudio de casos y controles, prospectivo en el tiempo, en 70 casos de endometriosis diagnosticados en Profamilia de Medellín en el periodo comprendido entre marzo y agosto de 1987. El diagnóstico de endometriosis se realizó por visión laparoscópica de lesiones endometriósicas y adherencias en la cavidad pélvica. Los casos se catalogaron según la extensión de las lesiones de acuerdo con la clasificación del American Fertility Society (39). Se establecieron cuatro grados de severidad, en una escala del I a IV. Todas las laparoscopias fueron realizadas por los cinco ginecólogos laparoscopistas de la institución. Se seleccionaron para la investigación únicamente los casos residentes en el área metropolitana de Medellín.

Por la falta de estudios previos, el tamaño de la muestra se estimó en 70 casos y se identificaron razones de disparidad de 3. Lo anterior puede limitar los resultados, en cuanto a que la detección de 
razones de disparidad bajas no siempre se podrán identificar con este estudio. Los controles comprendieron 3 grupos: un control vecino y dos controles institucionales que se buscaron en Profamilia y se denominaron control laparoscopia y control citología. Los controles con laparoscopia se identificaron el mismo día que se diagnosticó un caso y se escogieron aleatoriamente entre los pacientes con resultado negativo para endometriosis por laparoscopia. Los controles con citología se buscaron al día siguiente que se diagnostico un caso, entre las pacientes que acudieran a citología y llenaran los siguientes criterios: edad entre 15 y 45 años, con examen pélvico normal y sin historia de dolor pélvi co ni de dismenorrea. Para la selección del control vecino, la entrevistadora se desplazó hasta la casa de la paciente con endometriosis y siguiendo hacia la derecha buscó una vecina que llenara los siguientes requisitos: edad entre 15 y 45 años y sin historia de dolor pélvico ni de dismenorrea.

Las variables estudiadas fueron: edad, grupo étnico, nivel socio-económico, educación, ocupación, edad de la menarquía, características del ciclo menstrual, paridad, edad del primer embarazo, uso de anticonceptivos orales, uso de dispositivo intrauterino, uso de tampón, antecedentes de cirugía ginecológica, antecedentes familiares de endometriosis, hábito de cigarrillo y práctica de deporte. La información fué recolectada por diligenciamiento de encuestas. Las 280 encuestas fueron realizadas por una misma persona previamente entrenada sobre precisión en la aplicación de los términos y estandarización de las preguntas. Al realizar la encuesta a los casos y a los controles con laparoscopia, la entrevistadora no conocía si se trataba de una enferma o de un control. Con los otros controles no era posible mantener ciega la información.

Los datos se procesaron con ayuda de un microcomputador y mediante programa SPSS. Se calculó razón de disparidad (RD), ajustado por el método de Mantel y Haenszel y el chi cuadrado correspondiente. Se encontró asociación si RD era mayor de 1 y factor protector si RD era menor de 1 , valor de $\mathrm{P}$ significativo si $\mathrm{P}<$ de 0.05 . Se analizó por separado cada factor de riesgo, comparando los casos con los tres grupos diferentes de controles.

Al evaluar los resultados se tuvo en cuenta que, en la pareja Caso-control laparoscopia, el con- trol puede estar sobre equiparado por los factores sintomatología hipogástrica y nivel socio-económico. Caso-control citología: control que puede estar equiparado por las variables asociadas al uso del servicio de Profamilia y Caso-control vecino: control equiparado indirectamente por nivel socio-económico, al tomarse el control del mismo barrio.

Dentro de la investigación se definieron los siguientes términos:

Dismenorrea: menstruación dolorosa, con dolor intenso tipo cólico o calambre localizado en abdomen inferior y con irradiación a la espalda y a lo largo de los muslos. El dolor debe presentarse en varios ciclos consecutivos y comenzar horas antes o el mismo día del inicio del flujo menstrual. El dolor es tan intenso que obliga a buscar ayuda médica o lleva a la automedicación de analgésicos diversos.

Dolor pélvico: dolor localizado en la parte baja del abdomen, de por lo menos 6 meses de duración. Es un dolor crónico que aparece en cualquier momento del ciclo y su intensidad puede aumentar durante la menstruación y durante el acto sexual.

Hipermenorrea - polimenorrea: ciclo $<$ de 27 y duración $\geqslant 7$ días.

\section{RESULTADOS}

Los 70 casos de endometriosis representaron el $11.4 \%$ de las laparoscopias de Profamilia en el periodo de estudio. Entre los casos, la localización más común de las lesiones se detectó en los ligamentos útero-sacros con $48 \%$, peritoneo pélvico (Douglas y ligamento ancho) con 30\%, ovarios 20\% y trompas $2 \%$. En cuanto a la extensión de las lesiones, se encontró $68 \%$ grado I, $14 \%$ grado II, $12 \%$ grado III y $6 \%$ grado IV. El rango de edad de la mayoría de las pacientes con endometriosis osciló entre 20 y 40 años. Hubo treinta y tres pacientes, $47.1 \%$ con edad entre los 25 y los 32 años y éste último rango, se identificó como el grupo de edad de mayor riesgo para endometriosis en el estudio. Con respecto al grupo étnico se detectó que el $58.2 \%$ del total de las encuestadas era blanca y el resto $41.8 \%$ era mestiza. Solo hubo una paciente negra. Al observar la distribución de las dos razas predominantes en cada uno de los cuatro grupos estudiados, se encontró persistentemente un promedio de $57 \%$ pa- 
ra raza blanca y $43 \%$ para la raza mestiza. Esto, tal vez refleja el componente étnico de la población y hace pensar que no existe una raza de mayor riesgo para endometriosis en nuestro medio. Al evaluar el nivel socio-económico no se demostró ninguna asociación, porque los cuatro grupos estaban equiparados indirectamente en este parámetro. Una probable tendencia heredada a desarrollar endometriosis no se encontró, ya que no se logró demostrar antecedente familiar de endometriosis entre los casos. $\mathrm{Al}$ evaluar la paridad, se hallaron cuarenta y seis pacientes, $65.7 \%$, que no tenían embarazos, de las cuales quince, $21.4 \%$ acudieron a laparoscopia para estudio de infertilidad. Hubo tres pacientes con endometriosis que tuvieron su primer embarazo después de los treinta años de edad. Entre las 70 pacientes con endometriosis, hubo cuarenta y uno, $58.5 \%$ con instrucción $\geqslant 10$ años y veintiuno, $30 \%$ dentro del grupo profesional y administrador. En cuanto a características menstruales, se hallaron diecinueve, $27.1 \%$ pacientes con ciclo $<$ de 27 días y once, $15.7 \%$ con sangrado $\geqslant$ de 7 días. La edad temprana de la menarquia se encontró en catorce, $20 \%$ de los casos. Cuarenta y cuatro, $62 \%$, no utilizaban anovulatorios orales y cincuenta y tres, $75.7 \%$, no usaban dispositivo intrauterino.

Los resultados demuestran que el desarrollo de la enfermedad puede explicarse en asociación a las siguientes variables: ciclo menor de 27 días, no embarazos y no uso de anticonceptivos orales. Este hallazgo se repite al comparar los casos con los tres controles, con $\mathrm{P}<0.05$. Excepto en la variable ciclo menor de 27 días en la pareja caso-control citología con $\mathrm{P}<0.10$. En todos RD $>$ de 1 (Tabla 1 ). Otros factores que también resultaron asociados fueron: ocupación (profesional-administración), edad de la menarquia menor de 11 años, instrucción mayor de 5 años y mayor de 10 años. Estos hallazgos se encontraron en las parejas caso-control citología y caso-control vecino. En todas con $\mathrm{P}<0.05 \mathrm{y}$ RD $>$ de 1 (Tabla 2). Al evaluar el uso de dispositivo intrauterino (DIU) se encontró un significativo riesgo más bajo para endometriosis entre las usuarias de DIU. En otras palabras el DIU tiene efecto protector. El riesgo fué de $0.4(\mathrm{P}<0.10)$ de 0.06 $(\mathrm{P}<0.05)$ y de $0.2(\mathrm{P}<0.05)$ que corresponden respectivamente a los grupos caso-control laparoscopia, caso-control citología y caso-control vecino (Tabla 1).
TABLA 1

\section{VARIABLES ASOCIADAS EN LOS TRES TIPOS} DE CONTROLES

\begin{tabular}{|l|c|c|c|}
\hline \multicolumn{1}{|c|}{ Variable } & $\begin{array}{l}\text { Caso-control } \\
\text { laparoscopia } \\
\text { RD }\end{array}$ & $\begin{array}{l}\text { Caso-control } \\
\text { citologia } \\
\text { RD }\end{array}$ & $\begin{array}{l}\text { Caso-control } \\
\text { vecino } \\
\text { RD }\end{array}$ \\
\hline $\begin{array}{l}\text { Ciclo }<27 \\
\text { días }\end{array}$ & $* * 3.3$ & $* 2.2$ & $* * 4.8$ \\
\hline $\begin{array}{l}\text { No embarazos } \\
\text { No uso anti- } \\
\text { conceptivos }\end{array}$ & $* * 2.0$ & $* * 65.1$ & $* * 5.1$ \\
\hline $\begin{array}{l}\text { Usa o usó } \\
\text { DIU (Factor } \\
\text { protector) } \\
\text { RD }<1\end{array}$ & $* 0.4$ & $* * 4.8$ & $* * 2.1$ \\
\hline
\end{tabular}

RD: Razón Disparidad

TABLA 2

VARIABLES ASOCIADAS EN DOS TIPOS DE CONTROLES

\begin{tabular}{|l|c|c|c|}
\hline \multicolumn{1}{|c|}{ Variable } & $\begin{array}{l}\text { Caso-control } \\
\text { laparoscopia }\end{array}$ & $\begin{array}{l}\text { Caso-control } \\
\text { citología } \\
\text { RD }\end{array}$ & $\begin{array}{l}\text { Caso-control } \\
\text { vecino } \\
\text { RD }\end{array}$ \\
\hline $\begin{array}{l}\text { Instrucción } \\
>5 \text { años } \\
\begin{array}{l}\text { Instrucción } \\
>10 \text { años }\end{array}\end{array}$ & - & $* * 2.7$ & $*$ \\
\hline $\begin{array}{l}\text { Profesional- } \\
\text { administrador. }\end{array}$ & - & $* * 2.9$ & $* * 14.5$ \\
\hline $\begin{array}{l}\text { Menarquia } \\
<11 \text { años }\end{array}$ & - & $* * 17.2$ & $* * 3.2$ \\
\hline
\end{tabular}

$\mathrm{RD}$ : Razón Disparidad

Se detectó asociación con cirugía ginecológica, aunque solamente el comparar el grupo de casos con control vecino. Se dieron 4 casos en los cuales se diagnosticó endometriosis después de un procedimiento quirúrgico. Ellos fueron: un caso con endometriosis ovárica después de histerectomía abdominal; endometriosis en el muñón proximal de las trompas y endometriosis umbilical después de ligadura de trompas por laparoscopia; endometriosis en úterosacros y ovario izquierdo después de curetaje que produjo sinequias en el cérvix y un caso con endometriosis en úterosacros, ligamento ancho de- 
recho y ovario derecho después de cirugía pélvica para liberar adherencias.

No se logró identificar ninguna asociación al evaluar las variables: cigarrillo, práctica de deporte, anomalía uterina y familiares con endometriosis, excep to que al comparar caso-control laparoscopia, se encontró un riesgo relativo de $1.9(\mathrm{P}<0.10)$ para las pacientes que hacen actualmente deporte. Aunque el nivel de $\mathrm{P}$ en este caso es de baja significancia, no se sabe como explicar este resultado. Tampoco se encontró aumento de riesgo relacionado con la práctica higiénica de uso de tampones durante la menstruación.

Pero con respecto a esta variable, no se pueden sacar conclusiones en este estudio debido al bajo uso, ya que entre las 280 mujeres encuestadas sólo hubo 5 usuarias del tampón.

Se realizó análisis de regresión logística (40) entre las variables que resultaron asociadas al comparar los casos con los 3 controles. Las variables sometidas a este análisis fueron: ciclo menor de 27 días, no embarazos, no uso de anticonceptivos orales y uso del DIU y se encon tró que el no embarazo y el uso del DIU tuvieron la mayor asociación, pero este hallazgo no significa que estos factores sean necesariamente causales (Tabla 3). También se efectuó análisis de regresión logística, incluyendo las variables anteriores y los factores ocupación, ins-

TABLA 3

ANALISIS DE REGRESION LOGISTICA CON LAS VARIABLES ASOCIADAS EN LOS TRES TIPOS DE CONTROLES

\begin{tabular}{|c|c|c|c|}
\hline Variable & Coeficiente & $\begin{array}{l}\text { Razón } \\
\text { Disparidad }\end{array}$ & Z \\
\hline $\begin{array}{l}\text { Giclo menor } \\
27 \text { días }\end{array}$ & -0.0687636 & $0.93(0.68-1.27)$ & 0.44 \\
\hline No embarazo & -0.3601683 & $0.70(0.55-0.88)$ & $-2.99 *$ \\
\hline $\begin{array}{l}\text { No uso anti- } \\
\text { conceptivos } \\
\text { orales }\end{array}$ & 0.5392960 & $1.71(0.92-3.18)$ & 1.71 \\
\hline $\begin{array}{l}\text { Usó o usa } \\
\text { DIU }\end{array}$ & -0.9384132 & $0.39(0.20-0.74)$ & $-2.84 *$ \\
\hline
\end{tabular}

$Z \geqslant 1.96$

$* \mathrm{P}<0.05$ trucción y edad temprana de la menarquia y se repitió el mismo hallazgo para las variables no embarazos y uso de DIU (Tabla 4).

TABLA 4

\section{ANALISIS DE REGRESION LOGISTICA CON TODAS LAS VARIABLES ASOCIADAS}

\begin{tabular}{|l|c|c|c|}
\hline \multicolumn{1}{|c|}{ Variable } & Coeficiente & \multicolumn{1}{c|}{$\begin{array}{c}\text { Razón } \\
\text { disparidad }\end{array}$} & $\mathbf{Z}$ \\
\hline $\begin{array}{l}\text { Ciclo menor } \\
27 \text { días } \\
\text { No embarazos }\end{array}$ & -0.3448240 & $0.70(0.55-0.91)$ & $-2.69^{*}$ \\
$\begin{array}{l}\text { No usó anti- } \\
\text { concetivos } \\
\text { orales }\end{array}$ & 0.5724079 & $1.77(0.94-3.32)$ & 1.79 \\
$\begin{array}{l}\text { Usó o usa } \\
\text { DIU }\end{array}$ & -0.9361938 & $0.30(0.20-0.75)$ & $-2.81^{*}$ \\
$\begin{array}{l}\text { Instrucción } \\
>\quad 5 \text { años y } \\
>10 \text { años } \\
\begin{array}{l}\text { Profesionaly } \\
\text { administrador }\end{array}\end{array}$ & -0.1050900 & $0.90(0.77-1.04)$ & -1.38 \\
$\begin{array}{l}\text { Menarquia } \\
<11 \text { años }\end{array}$ & 0.0031536 & $1.00(0.86-1.16)$ & 0.04 \\
\hline
\end{tabular}

\section{DISCUSION}

Tradicionalmente se ha dicho que existe un mayor riesgo de endometriosis en la población de clase social alta y especialmente entre las mujeres que postergan el matrimonio y la maternidad (25). En este estudio no se demostró una mayor proporción de casos ubicados en los grupos de población que representaban los niveles socieconómicos más altos, pero se encontró una mayor proporción de casos en mujeres con un nivel de instrucción alto y ocupación dentro de los grupos profesional y administrador. Esta asociación puede explicarse por aumento del estrés, el cual produce secreción alterada catecolaminas que son las reguladoras de las hormonas hipotalámicas (41) o, estrés que puede llevar a hiperprolactinemia (17). Estas características pueden producir un desbalance hormonal y originar ciclos ovulatorios en los cuales la producción hormonal se desvía a favor del estrógeno y hay disminución en la producción de progesterona. En presencia de estas características, se cree, que se favorece el crecimiento de los implantes. Los factores educa- 
ción y ocupación también pueden reflejar aspectos que se relacionan con el estilo de vida como la baja paridad y demora para tener hijos. Estos últimos, a su vez, también se han descrito asociados al desarrollo de la entidad (25). Se observó que el $67 \%$ de las pacientes estaban en el grupo de edad de 21 a 35 años y el $47.1 \%$ se encontraba entre los 25 y 32 años. No se demostró que fueran de mayor riesgo los grupos considerados en la hipótesis con edad menor de 20 años y edad comprendida entre los 30 y 50 años y tampoco se vió correlación entre mayor edad y grado de severidad de la enfermedad.

Las variables que resultaron asociadas: ciclo menor de 27 días, no embarazos y edad temprana de la menarquia, pueden explicarse por la prolongada exposición a menstruación retrógrada y la aparición de un posible desbalance hormonal. Ante la evidencia de menstruación rètrógrada demostrada en el $90 \%$ de las mujeres con o sin endometriosis (42), se puede concluir que éste no es el único factor que determina la aparición de la entidad y, que otros factores como el hormonal o el inmunológico podrían estar implicados en el desarrollo de los implantes. Se sabe de la necesidad de un influjo hormonal para sostener los implantes ectópicos de la endometriosis $(11,15,43)$, pero no se sabe si es un imbalance hormonal el que inicia el proceso. Es posible que se requiera la presencia de una disfunción ovulatoria que se repita al menos durante varios ciclos para inducir el crecimiento de los focos. Esta afirmación puede tener correlación con los informes recientes de la literatura que demuestran coexistencia de la endometriosis con defectos en la fase lútea $(15,19)$, folículo lutenizado no roto $(9,20,21$, $22)$, hiperprolactinemia $(15,17,18)$ y aborto expontáneo (23). Pero el conocimiento de si es una disfunción ovulatoria la que inicia el proceso, o si son dichas alteraciones hormonales asociadas una consecuencia de la enfermedad, aún no está claro. Es posible que exista un mecanismo inmunológico que prevenga la formación de los implantes endometriales ectópicos. Pero quizás, en las pacientes que desarrollan la enfermedad, es te mecanismo inmunológico se encuentra disminuido o desgastado por el continuo y crónico reflujo endometrial o tal vez, este mecanismo inmunológico protector no existe en las mujeres que tiene alguna alteración específica en la inmunidad mediada por células $(9,13,14,15,16)$. La relación entre endometriosis e infertilidad es quizá la más crucial en la epidemiología de la enfermedad. La dificultad radica en poder definir si la baja paridad encontrada entre los casos es causal o es una consecuencia de la enfermedad. En este estudio se revisó la indicación para la laparoscopia en tre los casos y se encontró que quince pacientes, $21.4 \%$, fueron sometidas a laparoscopia para estudio de infertilidad, aunque cuarenta y seis pacientes $65.7 \%$ no tenían embarazos.

El no uso de anticonceptivos orales se encontró como factor asociado al desarrollo de la enfermedad. En otras palabras, el uso de anticonceptivos es un factor protector. Se explica, porque los anovulatorios llevan a la producción de pseudodecidua con formación de un endometrio no viable y además causan disminución del flujo menstrual, lo que en última instancia disminuye la exposición de la pelvis al contenido menstrual $(11,44)$. Se identificó el uso de dispositivo intrauterino como factor protector. Con el uso de DIU hay aumento de la síntesis de prostoglandinas endometriales, y es posible que estas al ser regurgitadas a la cavidad abdominal junto con la menstruación, impidan el crecimiento de los implantes. O tal vez, la reacción inflamatoria estéril que se produce en el endometrio en presencia del DIU, genera un ambiente citotóxico que inhibe la implantación del detrito menstrual regurgitado.

Este estudio epidemiológico indica que el riesgo de endometriosis puede relacionarse con características menstruales que permitan una mayor exposición de la pelvis al contenido menstrual, al tener mayor probabilidad de endometriosis las mujeres sin embarazos, con ciclos cortos y con una menarquia temprana. Además, los factores iden tificados como protectores, uso de dispositivo intrauterino y uso de anovulatorios orales, pueden también reforzar la teoría de la implantación si fuera correcto el mecanismo sugerido en relación a que el dispositvo intrauterino origine un ambiente inflamatorio en el endometrio que disminuya la posibilidad de implantación al ocurrir menstruación retrógrada y si los anticonceptivos orales conllevan a disminución del flujo menstrual. De acuerdo con lo anterior, se sugiere que la menstruación retrógrada es un factor desencadenante, pero no puede descartarse que un factor hormonal patológico o inmunológico participen en el proceso.

Trabajo financiado parcialmente por la Fundación Pedro Nel Cardona. 


\section{RESUMEN}

Se realizó un estudio prospectivo de casos y controles en Profamilia de Medellín. Se exploraron factores de riesgo para endometriosis en 70 casos diagnosticados por visión laparoscópica. Se compararon las diferentes variables entre los casos y los controles. Por cada caso se identificaron tres controles: un grupo de las pacientes sometidas a laparoscopia sin endometriosis, otro de la consulta de citología y un tercer grupo del vecindario del caso.

Los resultados demuestran que existe mayor riesgo para endometriosis en presencia de las variables: no embarazos, ciclo menor de 27 días, menarquia temprana, no uso de anticonceptivos orales, instrucción mayor de 5 años y mayor de 10 años, y en el grupo profesional y administrador. También se detectó un significativo más bajo riesgo para endometriosis asociado al uso de dispositivo intrauterino.

\section{SUMMARY}

A case-control prospective study of patients with diagnosis of endometriosis was conducted at Profamilia in Medellín, Colombia. Risk factors for endometriosis in 70 patients diagnosed by laparoscopy were explored; the controls were of three types: one, patients on whom no endometriosis was found at laparoscopy; two, patients who had come for a Pap test, and three, neighbors of the patient. Different variables were compared between the cases and their controls.
Our results show that there is a greater risk for endometriosis if the following variables are present: no pregnancy, cycles of less than 27 days, early menarche, no use of oral contraceptives, schooling of more than 5 and even better, 10 years, in the subgroup of professionals and administrators. A significant lower risk for endometriosis was also detected in patients fitted with an IUD.

\section{AGRADECIMIENTOS}

A los doctores Jaime Botero Uribe, GinecoObstetra, Profesor Emérito Universidad de Antioquia y Profesor Titular CES y Germán González E., Epidemiólogo, Jefe del Centro de Investigaciones de la Facultad Nacional de Salud. "Héctor Abad Gómez”, por su asesoría en la elaboración del proyecto y su valiosa ayuda en el procesamien to y análisis de los datos.

A Profamilia - Medellín y a su personal de enfermería por la colaboración prestada para la recolección de los casos y controles institucionales.

A la señora Leonor Camargo por su esfuerzo y esmero en la realización de las encuestas.

A la Fundación Pedro Nel Cardona por su aporte económico que hizo posible la ejecución de este estudio.

\section{BIBLIOGRAFIA}

1. Botero J.; CASTAÑEdA A.; GONZAlez G. Diagnóstico de Endometriosis en Medellín-Colombia. Revista Colombiana de Obstetricia y Ginecología. 1987; Vol 38 No. 5: 346-355.

2. SAMPSON, J.A. Peritoneal Endometriosis, due to menstrual dissemination of endometrial tissue into-peritoneal cavity. Am J. Obstet Ginecol 1927; 14: 422.

3. SAMPSON, J.A. Heterotopic or misplaced endometrial tissue. Am J. Obstet Ginecol 1925; 10: 649.

4. NOVAK, E. Pelvic Endometriosis. Am J. Obstet Gynecol 1931, 22: 826 .
5. OLIKER A.J.; HARRIS AE. Endometriosis of the bladder in a male patient. J. Urol 1971; 106: 858-59.

6. PINKERT TC; CATLON CE; STRAUSS R. Endometriosis of the urinary bladder in a man with prostatic carcinoma. Cancer 1979, 43: 1562-1567.

7. DOTY DW; GRUBER JS; GORDON CW et al. $46 \mathrm{XY}$ pure gonadal dysgenesis: report of 2 unusual cases. Obstet Gynecol $1980 ; 55$ (supplement 3 ): 61-63 S.

8. EL MAUgoub S; YASSEN S. A Positive proof for the Theory of celomic metaplasia. Am J. Obstet Gynecol 1980; 137 : $137-140$. 
9. DMOWSKI, P; RADWANSKA, E. Current concepts on Pathology, histogenesis and etiology of endometriosis. Acta obstétrica et ginecolg Scandinava. Supplement 1984; 123: 29-35.

10. RANNEY, B. Etiología, prevención e inhibición de la Endometriosis. Clin Obstet Gynecol 1980; 3: 893-901.

11. ROBERTSON WB. Nueva valoración del endometrio en la infertilidad Clin obstet Gynecol 1984 Apr; 11 (1): 209-225 .

12. HALBAN, J. Hysteroadenosis metastatica. Die Lymphogenese der Sog. Adenofibromatosis heterotópica. Arch Gynok 1925; 124: 457.

13. DMOWSKY Wp; STECLE RW; BAKER GR. Deficient cellular inmunity in endometriosis. Am J Obstet Gynecol 1981 Oct. $15 ; 141(4): 377-83$

14. BADAWY SZA; GUENCA V; STITZAL A; JACOBS RDB; THOMAS RH. Autoinmune phenomena in infertile patient with endometriosis. Obstet Gynecol 1984. 63: 271.

15. SCHMIDT, C. Endometriosis: a reappraisal of Pathogenesis and treatment. Fertil Steril 1985 Aug; 44 (2): 157-173.

16. BARBIERI RL; NILOFF JM; BAST RC; SCHAETZL E; KISTNER RW; KNAPPP RC. Elevated serum concentrations of CA-125 in patients with advanced endometriosis. Fertil Steril $1986 ; 45(5)$ : 630-34.

17. CHANG RJ. Hiperprolactinemia y alteraciones menstruales. Clin Obstet Gynecol 1983; 26 (3): 897-911.

18. HIRSCHOWITZ J.S.; SOLER NG; WORTSMAN J. The galactorrhea endometriosis Syndrome. Lancet, 1978. 1: 896.

19. HULL MGR. Ovulation Failure and induction. Clin Obstet Gynecol 1981. 8 (3): 753-785.

20. KONINCKX PR; IDE P; VANDENBROUEKE W; BROSENS IA. New aspects of the pathophysiology of endometriosis and associated infertility. J Reprod Med. 1980; 24: 257.

21. BROSENS I; KONINCKX P. BROECKX W. Endometriosis. Clin obstet Gynecol 1981, 8 (3): 639-651.

22. DONNEZ J; LANGEROCK S; THOMAS K. Peritoneal Fluid volume, 17 Beta-estradiol and progesterone concentrations in Women with Endometriosis and luteinized unruptured folicle syndrome. Obstetrical and Gynecological survey 1985, 39: 220.
23. OLIVE DL; FRANKLIN RR; GRATKINS L.V. The association between endometriosis and spontaneous abortion: a retrospective study. J. Reprod Med. 1982; 24: 333-338.

24. GONZALEZ GONZALEZ CF ; GONZALEZ GUTIERREZ JT; GOMEZ RUIZ VELASCO A. Endometriosis de la adolescencia. Ginecol obstet Mex 1984 Dec; 52 (332): 305-306.

25. HOUSTON De. Evidence For the risk of Pelvic endometriosis by age, race and socioeconomic status. Epidemiologic Reviews 1984; 6: 167-191.

26. OBERMEYER, CM; ARMENIAN, HK; AZOURY R; Endometriosis in Lebanon. American Journal of Epidemiology $1986 ; 124: 762-767$.

27. SIMPSON JL; ALIAS S; MALINAK LR; BUTTRAM VC. Heritable aspects of endometriosis. Am J Obstet Gynecol 1980 Jun 1: 137 (3): 327-37.

28. KEETEL, CT. Observations on the pathology and spread or endometriosis based on the theory of benign metastases. Am J. Obstet Gynecol 1951; 62: 477-478.

29. ZAMAH NM; DODSON MG; STEPHENS LC; BUTTRAM VC Jr. BESCH PK; KAUFMAN RH. Transplantation of normal and ectopic human endometrial tissue into athy mic nude mice. Am J. Obstet Gynecol 1984 Jul 15; 149 (6): 591-7.

30. VERNON M W; WILSON EA. Studies on the surgical induction of endometriosis in the rat. Fertil Steril 1985; $44(5)$ : 684-94.

31. VERMESH M; ZBELLA E; MENCHACA A; CONFINO E. LIPSHITZ S. Vesical endometriosis Following bladder injury. Am J Obstet Gynecol 1985; 153: 894-5.

32. DONNEZ J; CASANAS-ROUX F; FERIN J; THOMAS K. Tubal Polyps, epithelial incluions, and endometriosis after tubal sterilization. Fertil steril $1984 ; 41: 564-8$.

33. STOCK RJ; Postsalpingectomy Endometriosis: A Reassessment. Obstet Gynecol 1982; 60: 560-570.

34. FUNK, RL. Endometriosis Focus in the endocervix Following soon after conización. Pathologe 1984 march; 5 (2): 110-2.

35. SANTIFILIPPO J.S.; WAKIM NG; SCHIKLER KN; Y USSMAN MA. Endometriosis in association with uterine anomaly. Am J. Obstet Gynecol 1986; 154: 39-43.

36. GONZALEZ GONZALEZ CF; GONZALEZ GUTIERREZ JT; GOMEZ RUIZ VELASCO A. Endometriosis y Esterilidad. Gynecol Obstet Mex. 1984 Jun; 52 (326): 153-155. 
37. CRAMER, D; WILSON, E; STILLMAN; BERGER M.J.; BELISLE S. The relation of endometriosis to menstrual characteristics, smoking, and excercise. Jama 1986 Apr 11, 255 (14): 1904-8.

38. LAMB K, BERG N. Tampón use women with endometriosis. J. comunity Health 1985 Winter. 10 (4): 215-225.

39. AMERICAN FATILITY SOCIETY: Classification of endometriosis. Fertil-Steril. 1979. 32: 633.

40. MCGEE D. Epidemiologic Programs for computers and calculators. A program for logistic regression on the IBMPC. Am J. of epidemiology. 1987. 124 (4): 702-705.
41. YOUNGS DD; REAME N. Aspectos psicosomáticos de la disfunción menstrual. Clin Obstet Gynecol 1983; 26 (3): 951-959.

42. HALME J;HAMMOND MG; HULKA JF; RAJ SG; TALBERT LM. Retrograde menstruation in Healty women and in patients with endometriosis. Obstet Gynecol 1984; 64: 151-154.

43. SCHWEPPE KW, WYNN RM. Endocrine dependency of endometriosis: An ultrastructural Study. Obstetrical and Gynecological survey. 1985. 39: 714 .

44. DAWOOD MY. Dismenorrea. Clin Obstet Gynecol 1983; 26 (3): 875-885.

\section{SUSCRIPCIONES}

Para todo lo relacionado con suscripciones, correspondencia, canje, etc., dirigirse a REVISTA COLOMBIANA DE OBSTETRICA Y GINECOLOGICA, Bogotá, D.E. Colombia. Carrera 23 No. 39-82, Piso 2o. Apartados Aéreos: 14961 y 34188. Teléfono: 2681485.

Dirección comercial PUBLIMEDICAS LTDA. Calle 72 No. 6-44 Piso 11. Teléfono: 2358358, Bogotá, D.E.

Suscripción anual (4 Números), No. Socios $\$ 3.600 .00$ (moneda colombiana) o US\$50.oo, para exterior - otros. Valor del ejemplar $\$ 1.000 .00$ (moneda colombiana). 